\title{
Achieving better health for people with intellectual disability: the power of policy
}

Rory Sheehan, Yona Lunsky and Angela Hassiotis

\section{Summary}

People with intellectual disability have high mental and physical healthcare needs, which must be addressed on individual, local and national levels. Policy interventions informed by research and stakeholder views and extending beyond a focus on health are needed to reduce inequities in this group.

\section{Declaration of interest}

None.

\section{Copyright and usage}

(c) The Royal College of Psychiatrists 2018. This is an Open Access article, distributed under the terms of the Creative Commons Attribution-NonCommercial-NoDerivatives licence (http://creativecommons.org/licenses/by-nc-nd/4.0/), which permits noncommercial re-use, distribution, and reproduction in any medium, provided the original work is unaltered and is properly cited. The written permission of Cambridge University Press must be obtained for commercial re-use or in order to create a derivative work.
Rory Sheehan (pictured) is a National Institute for Health Research Doctoral Research Fellow in the Division of Psychiatry at University College London. He researches health interventions for people with intellectual disability, in particular the use of psychotropic medication. Yona Lunsky is a Professor at the Department of Psychiatry, University of Toronto, and Senior Scientist, at the Centre for Addiction and Mental Health, studying intellectual disabilities and mental health. Angela Hassiotis is a clinical academic in the Division of Psychiatry at University College London and the Camden Learning Disability Service. Her main interests are in mental health services research in people with intellectual and developmental disabilities across the lifespan.

Despite difficulties in recognition and accurate diagnosis, it is now well established that the rate of mental disorder in people with intellectual disability is similar to or exceeds that of the general population. ${ }^{1}$ The recent publication in this journal of a study reporting the prevalence of mental illness and its association with mental health conditions in people with intellectual disability is a timely reminder of the additional mental and physical health needs of this group. ${ }^{2}$ The authors use data collected in the 2011 Scottish census, thereby achieving almost universal population coverage, demonstrating once again the power of exploiting routinely collected data in health research. The analysis showed that people with intellectual disability were seven times more likely to self-report (or their caregiver to proxy-report) a current mental health condition than those in the comparison group without intellectual disability. This apparent higher rate of mental ill health exists across the lifespan and is associated with significant additional personal, social and economic costs. The authors highlight the relative paucity of research evidence for effective psychotherapeutic interventions in this group and highlight the continuing need to improve individual outcomes and quality of life. Alongside individual actions, however, wider strategies to promote well-being and deliver a consistent response to ill health are necessary. National policy is an important lever for directing and effecting change.

\section{Policy for intellectual disability}

Over the past 40 years, successive waves of health and social care policy, most aimed at promoting social inclusion and the use of mainstream health services, have changed the landscape of services for people with intellectual disability. Transforming Care is the current flagship UK government policy to improve care for people with intellectual disability and mental illness or challenging behaviour. ${ }^{3}$ In a spirit of co-production, the priorities of Transforming Care have been shaped by people with intellectual disability and their supporters. A central aim of the programme is further substantial reduction of specialist intellectual disability assessment and treatment in-patient beds for people with challenging behaviour in the absence of mental illness, accompanied by increased investment to improve the capacity and quality of community services. Also prominent in Transforming Care is a commitment to reducing the over-reliance on psychotropic drugs in people with intellectual disability and, in particular, the use of antipsychotic drugs for challenging behaviour. ${ }^{4}$ Concern about the overuse of psychotropic medication in this group is not new, nor is it confined to the UK, and attempts to reduce prescribing are underway in several countries. Finally, there needs to be a programme of research to develop and test the clinical and cost-effectiveness of interventions for mental disorders in this population. This is singularly lacking at present, with evidence for many pharmacological and psychosocial interventions being supported only by feasibility studies.

People with intellectual disability present with multi-morbidity, ${ }^{5}$ and general health, physical disability and mental ill health in this group are closely associated and must be considered alongside one another. ${ }^{1}$ Certain health problems that are common in people with intellectual disability, particularly overweight and obesity, can be exacerbated by long-term use of psychotropic medication, underlining the importance of close physical health monitoring and regular psychotropic medication review. However, people with intellectual disability frequently report difficulties accessing health services and, despite increases in life expectancy over the past several decades, experience high rates of premature death. ${ }^{6-8}$ Health promotion activities and screening programmes can often fail to reach the most vulnerable, including those with intellectual disability.

The annual primary care health check for people with intellectual disabilities, introduced in England in 2008, is one means by which mental and physical health issues can be proactively identified and, if necessary, treated early. Evidence has accumulated from the UK and elsewhere that regular comprehensive health checks are of benefit in identifying unmet health needs and reducing barriers in access to health services. ${ }^{9}$ Although it seems reasonable to presume that a programme of health checks will yield longer-term health 
benefits, further research is needed to fully understand the effect of regular health checks on outcomes and resource use over time.

\section{Policy evaluation}

The full terms by which Transforming Care will be judged are still to be determined, but its effects should include longer, healthier and more fulfilling lives for people with intellectual disability. Evaluating the success or otherwise of health policy can be difficult, but it is vital if maximum benefit is to be achieved. Policy outcomes are often farreaching, multidimensional and difficult to measure: effects may develop slowly and only become visible after a considerable time, and in an uncontrolled environment it can be difficult to determine a causal relationship between a policy intervention and subsequent events. Evaluation of the Transforming Care programme presents many of these challenges. Indicator measures include the number of people with intellectual disability admitted to mental health or assessment and treatment units, and the extent of potentially inappropriate psychotropic drug prescribing. In both cases, routinely collected data can be used to monitor progress, with linkage between data-sets providing an opportunity to assess broader impacts. Patient reported outcome measures that reflect the concerns and priorities of patients with intellectual disability and their families need to be incorporated into standard practice and reported nationally to drive service improvements.

Health policies may have unintentional consequences which might only be revealed through a comprehensive evaluation process; this risk is increased if they are developed without appropriate stakeholder consultation and application of available evidence. Reducing one drug class may merely increase prescribing of others; for example, there is some evidence that a drive to reduce the use of antipsychotic drugs in older adults with dementia has been associated with a corresponding increase in the use of antidepressants and other psychotropic drugs. ${ }^{10}$ Some have expressed concern that hurried bed closures as part of Transforming Care may increase the number of people with intellectual disabilities and offending behaviours who go to prison owing to lack of alternative forensic psychiatric services, or result in greater and longer-lasting restrictions through use of Deprivation of Liberty orders if they remain in the community. ${ }^{11}$

\section{A 'Health in All Policies' approach}

Poverty, unemployment, poor housing, social isolation, experience of abuse and discrimination are risk factors for ill health that are overrepresented in people with intellectual disability and contribute to the health inequities they experience. Supporting health and achieving parity for people with intellectual disability therefore requires that these broader determinants of health and social disadvantages be addressed. A 'Health in All Policies' approach, as advocated by the World Health Organization, argues for health considerations to be incorporated into decision-making across sectors, for example, in transport or town planning policy. Such an approach recognises the influence of economic and social context on health, and the differential effects that a policy may have on diverse groups, which could be particularly beneficial for those in vulnerable groups.

Widespread austerity measures and welfare reform have been disproportionately disadvantageous to people with disabilities, including those with intellectual disabilities, and may mitigate the benefits of (or indeed conflict with) policies taking a longer-term population perspective. ${ }^{12}$ Clinicians can play an important part in advocacy and ensuring that decision makers are informed of the health and equity consequences of policy options.

\section{Conclusions}

People with intellectual disability experience health problems that must be addressed both individually and through coherent national policy initiatives, both targeted and for the general population. Active involvement of people with intellectual disability and their families and carers is necessary to ensure that policies reflect reallife concerns.

Improving mental and physical health and reducing inequalities will only be realised where broader social and economic determinants of health are also considered; there is a risk that incremental gains achieved through targeted interventions might be undermined by a wider political agenda and competing priorities. A Health in All Policies approach should be considered as a way forward.

Rory Sheehan, BSC, MBChB, MSC, MRCPsych, Division of Psychiatry, University College London, UK; Yona Lunsky, PhD, C. Psych, Department of Psychiatry, University of

Toronto, and Centre for Addiction and Mental Health, Ontario, Canada;

Angela Hassiotis, MBBS, MA, PhD, FRCPsych, Division of Psychiatry, University College London and Camden Learning Disability Service, London, UK

Correspondence: Rory Sheehan, Division of Psychiatry, University College London, Maple House, 149 Tottenham Court Road, London, WIT 7NF. Email: r.sheehan@ucl.ac.uk

First received 18 Oct 2017, final revision 22 Nov 2017, accepted 29 Nov 2017

\section{References}

1 Cooper S-A, Smiley E, Morrison J, Williamson A, Allan L. Mental ill-health in adults with intellectual disabilities: prevalence and associated factors. $\mathrm{Br} J$ Psychiatry 2007; 190: 27-35

2 Hughes-McCormack LA, Rydzewska E, Henderson A, Macintyre C, Rintoul J, Cooper S-A. Prevalence of mental health conditions and relationship with general health in a whole-country population of people with intellectual disabilities compared with the general population. BJPsych Open 2017; 3: 243-8.

3 Department of Health. Transforming Care: A National Response to Winterbourne View Hospital. DH, 2012.

4 Sheehan R, Hassiotis A, Walters K, Osborn D, Strydom A, Horsfall L. Mental illness, challenging behaviour, and psychotropic drug prescribing in people with intellectual disability: UK population based cohort study. BMJ 2015; 351: h4326.

5 Cooper S-A, McLean G, Guthrie B, McConnachie A, Mercer S, Sullivan F, et al. Multiple physical and mental health comorbidity in adults with intellectual disabilities: population-based cross-sectional analysis. BMC Fam Pract 2015; 16: 110.

6 NHS Digital. Health and Care of People with Learning Disabilities. NHS Digital 2016.

7 Trollor J, Srasuebkul P, Xu H, Howlett S. Cause of death and potentially avoidable deaths in Australian adults with intellectual disability using retrospective linked data. BMJ Open 2017; 7: e013489.

8 Heslop P, Blair PS, Fleming P, Hoghton M, Marriott A, Russ L. The Confidential Inquiry into premature deaths of people with intellectual disabilities in the UK: a population-based study. Lancet 2014; 383: 889-95.

9 Robertson J, Roberts H, Emerson E, Turner S, Greig R. The impact of health checks for people with intellectual disabilities: a systematic review of evidence. J Intellect Dev Disabil 2014; 55: 1009-19.

10 Martinez C, Jones RW, Rietbrock S. Trends in the prevalence of antipsychotic drug use among patients with Alzheimer's disease and other dementias including those treated with antidementia drugs in the community in the UK: a cohort study. BMJ Open 2013; 3: e002080.

11 Taylor JL, McKinnon I, Thorpe I, Gillmer BT. The impact of transforming care on the care and safety of patients with intellectual disabilities and forensic needs. BJPsych Bull 2017; 41: 205-8.

12 British Medical Association. Health in all Policies: Health, Austerity and Welfare Reform. BMA, 2016 